\title{
The Role of Double Modality Ultrasonographic and Fluoroscopic Guided Superior Hypogastric Plexus Neurolysis in Treating Intractable Pelvic Cancer Pain: A Comparative Study
}

\author{
Ekramy M Abdelghafar' \\ Ahmed H Othman ${ }^{2}$ \\ Mahmoud Salem Soliman ${ }^{3}$ \\ Ayman Kilany ${ }^{4}$ \\ Mohammed H Shaaban ${ }^{5}$ \\ Ehab H Shaker (D) \\ 'Department of Anesthesia, ICU And \\ Pain Relief, National Cancer Institute, \\ Cairo University, Cairo, Egypt; \\ ${ }^{2}$ Department of Anesthesia, ICU and Pain \\ Relief, South Egypt Cancer Institute, \\ Assiut University, Assiut, Egypt; \\ ${ }^{3}$ Department of Anesthesia, Critical Care \\ and Pain Management, Faculty of \\ Medicine, Cairo University, Cairo, Egypt; \\ ${ }^{4}$ Department of Research on Children \\ with Special Needs, National Research \\ Center, Cairo, Egypt; ${ }^{5}$ Department of \\ Diagnostic \& Interventional Radiology, \\ Faculty of Medicine, Cairo University, \\ Cairo, Egypt
}

Background: Superior hypogastric neurolytic block is performed to block visceral pelvic pain. This could be performed through the anterior approach guided by CT or ultrasound and through a posterior approach, guided by fluoroscopy or CT.

Methods: Sixty adult patients with severe visceral pelvic pain (VAS>70 mm) were randomly divided into two groups. Group S: SHP block was done ultrasound guided using the anterior approach and confirmed by fluoroscopy. Group F: SHP block was done fluoroscopic guided using the posterior oblique approach. The VAS (visual analog scale), duration of the technique, time of X-ray exposure, patient satisfaction score, patient global impression of change (PGIC), quality of life score, and daily morphine consumption (mg/day) were measured pre-procedure and at the $1 \mathrm{st}, 4 \mathrm{th}, 8 \mathrm{th}$, and 12th week after the procedure. In addition, any side effects of the procedure were recorded.

Results: There was a significant difference in VAS between the two groups $(P<0.01)$ (better in group S). The quality of life score was improved from the pre-procedure in both groups $(P<0.05)$, and morphine consumption was significantly lower in group $\mathrm{S}$ than in group $\mathrm{F}(P<0.05)$ at the $1 \mathrm{st}, 4 \mathrm{th}$, and 8 th week and not significant at the 12 th week. The two groups show a statistically significant difference as regards the duration of the procedure and $\mathrm{X}$-ray exposure $(P<0.01)$. There was a statistically significant difference in the satisfactory score between the two groups at the 1st, 4th, 8th, and 12th week $(P<0.01)$. As regards the PGIC score, there was no statistically significant difference between the two groups $(P>0.05)$. In group $\mathrm{S}$, no back pain was reported, while 11 patients of group $\mathrm{F}$ complained from post-procedure back pain $(P<0.001)$.

Conclusion: The anterior ultrasound guided SHPB aided by fluoroscopy is suggested to be more superior to the standard fluoroscopic guided technique in relieving pelvic cancer pain and decreasing morphine consumption.

Keywords: superior hypogastric block, pelvic pain, fluoroscopy, ultrasound

\section{Introduction}

Pelvic pain is one of the poorly defined conditions that originate from complex pathophysiological pain generators in pelvic organs of the gastrointestinal, urogenital, nervous, or musculoskeletal systems. ${ }^{1}$ Around 15 million patients suffering from pelvic pain are managed by different medical specialties over time. $^{2}$
Correspondence: Ehab H Shaker

Department of Anesthesia and Pain Relief, National Cancer Institute, Cairo

University, Cairo, Egypt

Tel +2-01222438820

Email ehabhanafy2006@yahoo.com 
Pain is a frequent and handicapping feature in patients suffering from advanced pelvic malignancies. Neurolytic pelvic sympathectomy with chemicals (alcohol, or phenol) is considered a safe and effective solution for those patients who do not respond well to medical treatment with opioids or those who cannot tolerate opioids' adverse effects. $^{3,4}$

The superior hypogastric plexus (SHP) is one of the paravertebral sympathetic ganglia, located in the lower border of the L5 vertebra and upper part of the sacrum in the retroperitoneal space. It is considered as a continuity of the celiac plexus and the lumbar sympathetic ganglia. It is related to the bifurcation of the aorta and the ureters. The SHP has a sympathetic connection (both efferent and afferent fibers) with splanchnic nerves and aortic plexus. It innervates the viscera of the pelvis, including the urinary bladder, ureters, sigmoid colon down to the anal canal, and upper vagina. $^{5}$

Superior hypogastric neurolytic block is performed to block visceral pelvic pain, due to pelvic malignant pain, interstitial cystitis, pelvic inflammatory disease (PID), and endometriosis. This could be performed anteriorly aided by CT scan or ultrasonography or posteriorly, guided by fluoroscopy or CT scan., ${ }^{6,7}$

In the current study, we compared the classic fluoroscopic technique versus double means of guiding modality at the same time including both ultrasonography and fluoroscopy for superior hypogastric plexus phenol neurolysis in intractable pelvic pain in patients with cancer.

\section{Patients and Methods}

The local ethical committee (IRB No: 201516012.2) approved this study (Institutional Review Board of the National Cancer Institute - Cairo - Egypt) and written informed consent was taken from each patient after detailed explanation of the procedure benefits and adverse effects. The study was registered prospectively at ClinicalTrials.gov NCT03606811 and conducted in accordance with the Declaration of Helsinki. All of the study was done at the pain clinic and operation theater of the National Cancer Institute, Cairo University in the period from August 2018 to August 2019. The authors do not intend to share any data besides what are included in the manuscript and no further data will be shared.

Sixty patients with advanced or recurrent cancer of the bladder not responding to the initial medical (opioids or adjuvant pharmacotherapy or showed intolerable side effects) or radiotherapy treatment and suffering from severe visceral pelvic pain were enrolled in this study.

A consultant interventional radiologist performed the initial U/S technique and needle placement while any needle readjustment following the fluoroscopic visualization, contrast and neurolytic injections were performed by the consultant interventional pain physicians.

\section{Inclusion Criteria}

Patients $>18$ years of age with severe visceral pelvic pain (VAS $>70 \mathrm{~mm}$ ), and the pain was either refractory to medical treatment or the side effects of the drugs were not tolerated by the patients.

\section{Exclusion Criteria}

Patient refusal, coagulation disorders, local or systemic sepsis, previous neurological or psychiatric disorders, history of addiction, distorted local anatomy, advanced respiratory or cardiovascular disease, and those known to have allergy to the used medication.

All patients were instructed to fast for 8 hours (clear fluids were allowed for 4 hours) before the procedure. To identify the urinary bladder at the beginning of the procedure, the interventional radiologist performed a pelvic ultrasound to scan the pelvic structures and identify the urinary bladder, which is in a suprapubic position with some residual urine (remaining in spite of full voiding) and then color Doppler on the urinary bladder will show the so-called urinary jet which is the urine coming from the ureter to the bladder at the vesico-ureteric junction.

In the operating room, the patients were monitored with ASA-standard monitors (NIBP, pulse-oximetry, and ECG). An IV line was inserted, and oxygen was supplied through a nasal cannula. The patients were sedated using fentanyl 0.5 $\mu \mathrm{g} / \mathrm{kg}$ and midazolam $0.05 \mathrm{mg} / \mathrm{kg}$. A computerized random list was prepared, and the patients were randomly allocated into two groups:

Group S: included 30 patients where SHP block was done ultrasound guided using the anterior approach and confirmed by fluoroscopy.

Group F: included 30 patients where SHP block was done fluoroscopic guided using the posterior oblique approach.

\section{Ultrasound Guided Technique ${ }^{7}$}

For all patients, bowel preparation was done with bisacodyl and activated charcoal and all patients were instructed to empty their bladder before starting the procedure. The technique was done under complete aseptic conditions with the 
patient in the supine position. The division of the abdominal aorta into its two common iliac arteries was identified by using the curved ultrasound transducer (Sonosite, M-Turbo, USA) placed longitudinally, and then the body of the fifth lumbar vertebra was identified by rotating the transducer transversely (Figure 1). Local anesthetic infiltration using lidocaine $1 \%$ was injected at the entry site (skin and subcutaneous tissue) $1-1.5$ inches below the umbilicus. A 22-G, $15-\mathrm{cm}$ Chiba needle was introduced in an out of plane technique, and advanced away from vascular structures (using Doppler mode) until bony contact with the L5 vertebra (Figure 2). The needle then was withdrawn for $2 \mathrm{~mm}$ to avoid injection into the periosteum. Then, $3 \mathrm{~mL}$ contrast medium (iohexol-omnipaque $300 \mathrm{mg}$ iodine $/ \mathrm{mL}$, Nycomed, Ireland) was injected followed by antero-posterior and lateral fluoroscopic image to confirm the distribution of the dye. The

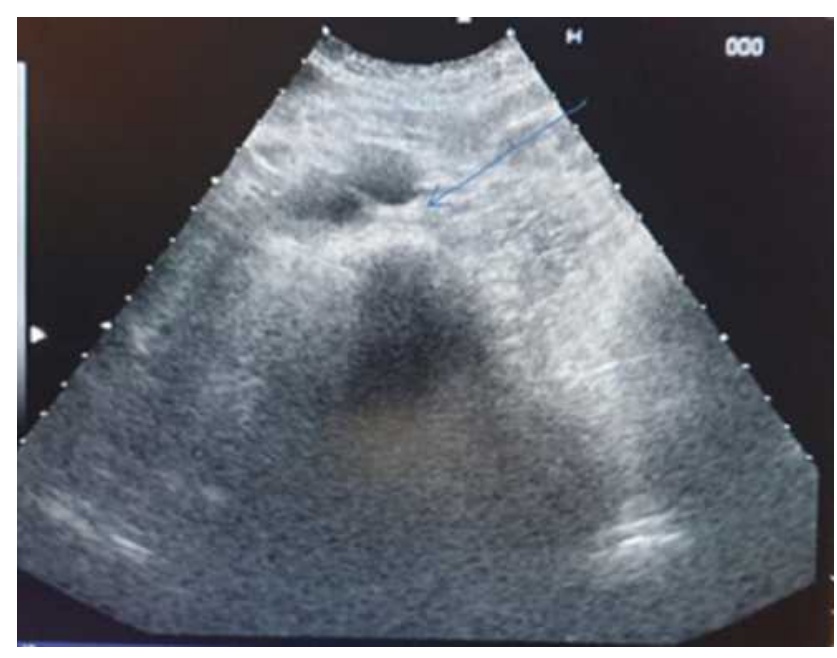

Figure I Ultrasonographic picture with the blue arrow pointing to L5 vertebrae and iliac vessels.

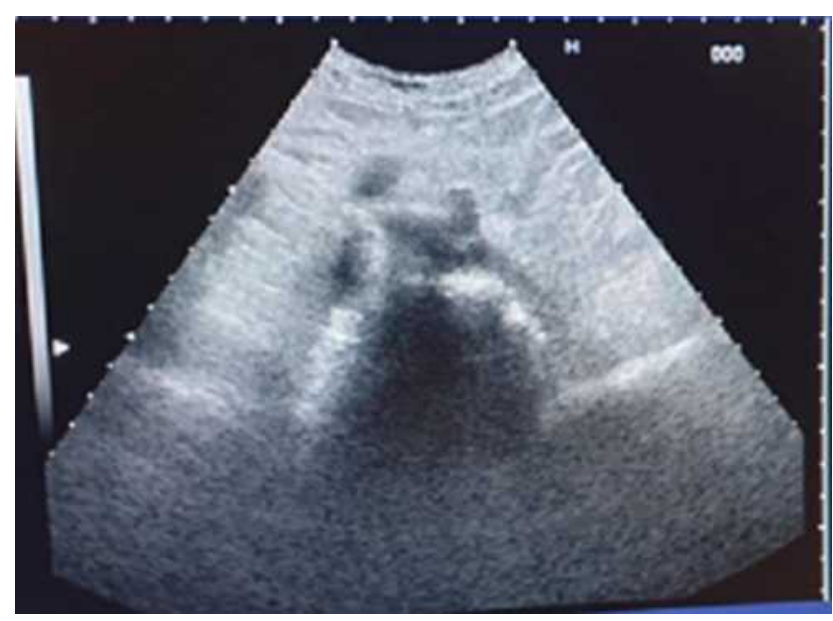

Figure 2 The needle in front of L5 out of plain technique. ultrasound probe was applied again and $8 \mathrm{~mL}$ of $8 \%$ phenol in saline was injected and the uniform distribution was confirmed under real-time sonography. Then, $0.2 \mathrm{~mL}$ lidocaine $1 \%$ was injected and the needle was removed (to avoid fistula formation).

\section{Fluoroscopic Guided Oblique Technique ${ }^{8}$}

The patient was placed in a prone position with a pillow under the pelvis to flatten the lumbar lordosis. After steep and cephalic orientation of the c-arm to straighten, the lower border of L5 was in the P/A view. Then, the c-arm was oriented $20-25^{\circ}$ obliquely and the entry point was located at the lower lateral border of L5. Local anesthesia was injected using 1\% lidocaine at the site of entry. A 15$\mathrm{cm}$ 22-G Chiba needle was inserted in the direction of the irradiation beam (end on, or the gun barrel technique). The Chiba needle was manipulated until a reasonable depth $(7-8 \mathrm{~cm})$, the dead lateral view taken, and then the needle is more inserted anterior to the psoas fascia (Figure 3). The procedure was repeated on the other side. Then $1-2 \mathrm{~mL}$ of contrast medium (iohexol-omnipaque $300 \mathrm{mg}$ iodine $/ \mathrm{mL}$, Nycomed, Ireland) was injected after negative aspiration. It should outline smoothly the L5-S1 junction both up and down without posterior spread toward the nerve root. The postero-anterior view was taken to confirm favorable dye spread (paramedian globular at L5-S1) (Figures 4 and 5). Then, $4 \mathrm{~mL}$ of $8 \%$ phenol in saline was injected in each needle and $0.2 \mathrm{~mL}$ of lidocaine injected during needle removal.

All patients were transferred to the recovery unit where their pain intensity and hemodynamic variables were assessed for 2 hours and were then discharged.

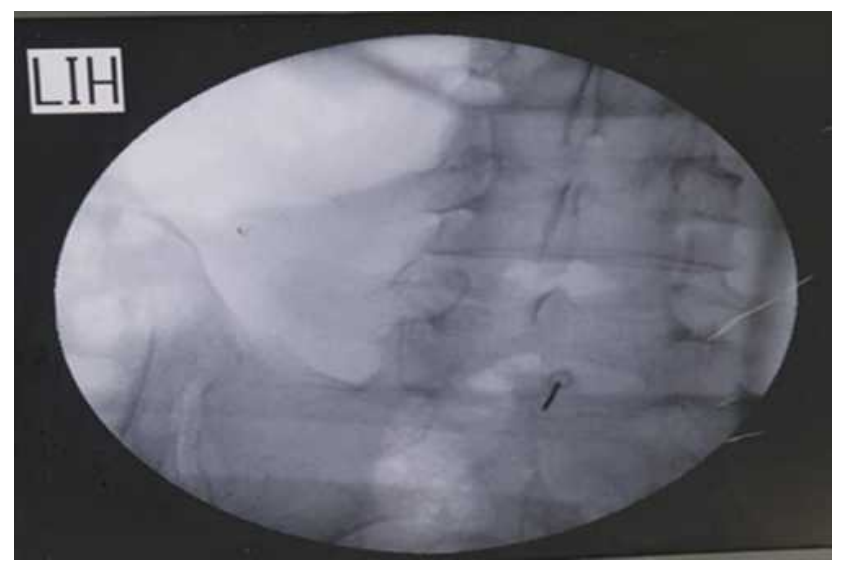

Figure $3 \mathrm{~A} / \mathrm{P}$ view with the needle at L5 fluoroscopic guidance. 


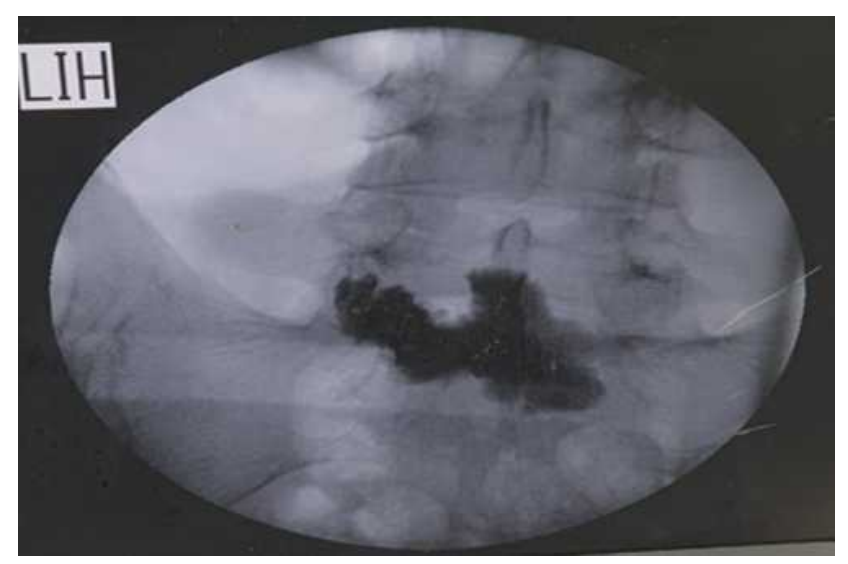

Figure 4 Distribution of the dye at AP view fluoroscopic guidance.

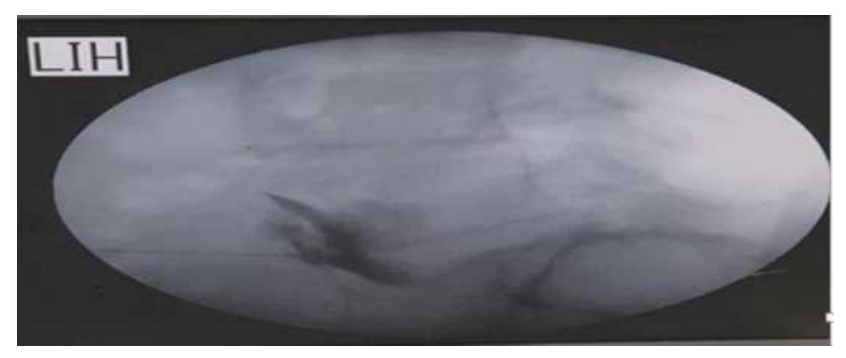

Figure 5 Spread of the dye in lateral view fluoroscopic guidance.

Data were recorded by a junior pain resident who was blinded to the study protocol, patient enrollment, and technique. The primary end point was VAS assessment (visual analog scale), a $100 \mathrm{~mm}$ scale with left end 0 means no pain and right end 100 means worst imaginable pain. It was measured pre-procedure and at the $1 \mathrm{st}, 4 \mathrm{th}$, 8th, and 12th week after the procedure.

Secondary end points included: duration of the technique, duration of $\mathrm{X}$-ray exposure, satisfactory score $(0=$ dissatisfied, $10=$ very satisfied $),{ }^{9}$ patient global impression of change (PGIC), ${ }^{10}$ quality of life score ${ }^{11}$ and daily morphine consumption (mg/day) at pre-procedure and the 1st, 4th, 8th, and 12th week after the procedure. In addition, any side effects of the procedure were detected and recorded (hypotension, nerve injury, needle discomfort, position discomfort, and urinary retention).

\section{Sample Size}

Based on a similar study done by Kamel et al ${ }^{12}$ showing the efficacy of an ultrasound guided anterior approach for superior hypogastric plexus neurolysis based on the VAS score measured before the procedure and after 3 months in either group: significant level or probability of type 1 error $=0.05$, power of the test statistics to be $90 \%$, expected within-group standard deviation of 1.5 , a critical difference of 2.9 (drop in VAS score), and ratio of sample size Group $2_{2}$

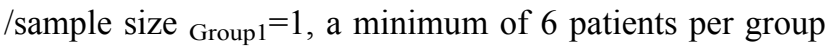
with a total of 12 patients are enough to see that effect.

Sample size can be increased up to 30 patients for each group to compensate for the number of losses in case of patients' loss to follow up and expectation that variables may not follow a normal distribution.

\section{Statistical Analysis}

Data will be described as mean $\pm \mathrm{SD}$ or as frequencies (number and percentages) when appropriate. Comparison of numerical variables between two study groups was carried out using parametric and non-parametric $t$-tests for independent samples. Within-group comparison of numerical variables was carried out using repeated measures ANOVA. $P$ values of less than 0.05 will be statistically significant. All statistical calculations are performed using statistical package for the social sciences (SPSS version, SPSS Inc., Chicago, IL, USA)

\section{Results}

Eighty-six patients were primarily assessed for eligibility, 17 were initially excluded ( 8 did not meet the inclusion criteria, 6 declined to participated, and 3 hesitated and were not fully convinced by the study). After randomization and allocation 5 patients were excluded from Group $\mathrm{S}$ (4 lost to follow up and 1 discontinued the protocol) while 4 patients were excluded from Group F (2 lost to follow up and 2 discontinued the protocol) (Figure 6).

There were no statistically significant differences between the two groups regarding demographic data $(P>0.05)$ (Table 1).

There was no statistically significant difference between the two groups as regards the pre-procedure VAS ( $P=0.983)$; however, there was a statistically significant difference in VAS between the two groups at the 1st, 4th, and 8th week $(P<0.01)$ whereas at the 12 th week there was no significant difference $(P=0.122)$. The decline in VAS score from the pre-procedure values was statistically significant in both groups and more significant in group S $(P<0.01)$ (Table 2).

The two groups show a statistically significant difference as regards duration of the procedure (minutes) and $\mathrm{X}$-ray exposure (seconds) $(P<0.01)$ (Table 3$)$.

There was no statistically significant difference between the two groups regarding the pre-procedure satisfactory score $(P=0.909)$. However, there was a statistically 


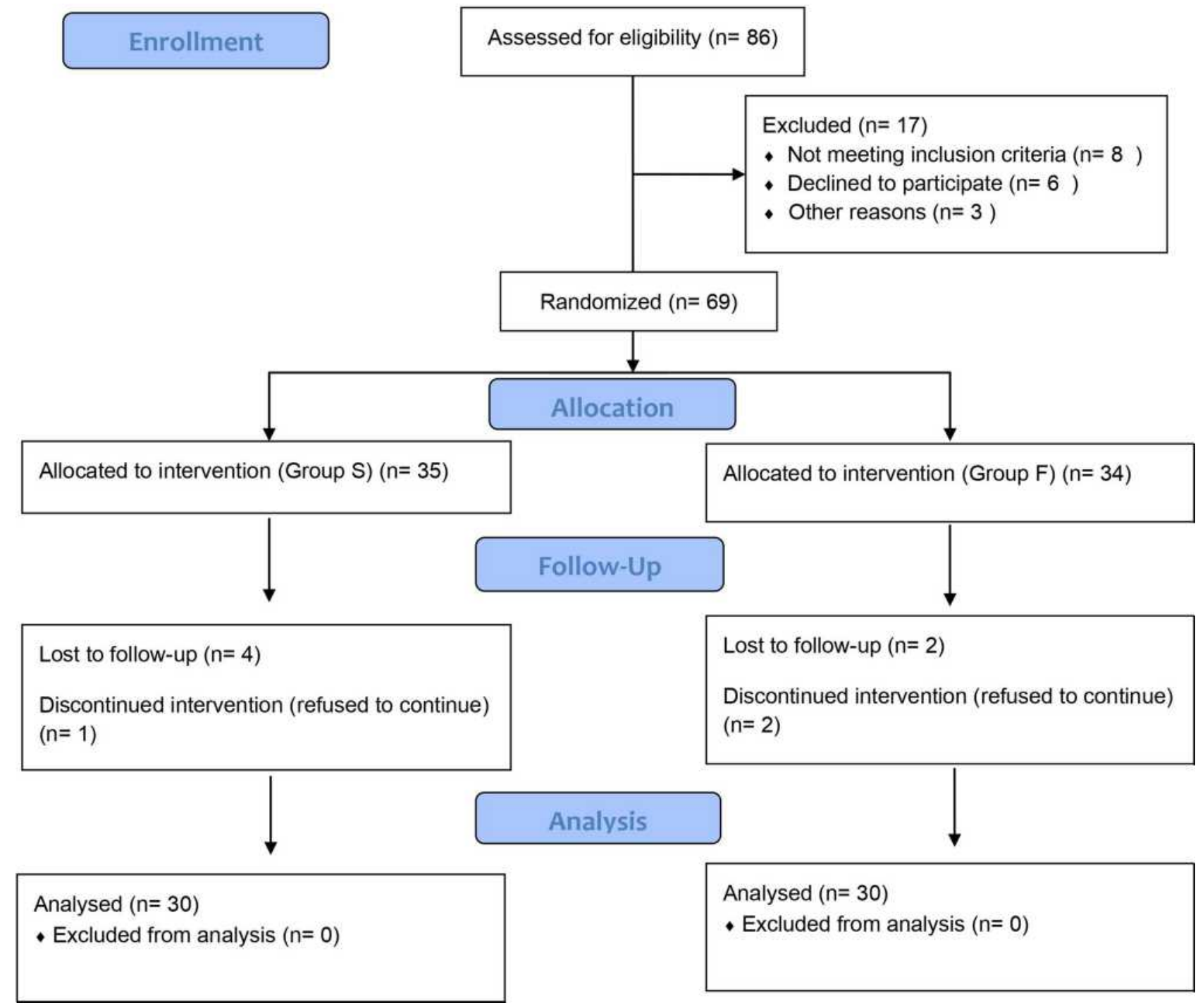

Figure 6 Consort flow chart.

significant difference in satisfactory score between the two groups at the $1 \mathrm{st}, 4$ th, 8 th, and 12 th week $(P<0.01)$. The improvement in satisfactory score from the pre-procedure values was statistically significant in both groups and more significant in group $\mathrm{S}(P<0.01)$ (Table 4$)$.

Table I Demographic Data

\begin{tabular}{|c|c|c|c|}
\hline & $\begin{array}{l}\text { Group S } \\
\text { (Mean士SD) }\end{array}$ & $\begin{array}{l}\text { Group F } \\
\text { (Mean士SD) }\end{array}$ & $P$ value \\
\hline Age & $55 \pm 9.33$ & $56.17 \pm 8.8$ & 0.92 \\
\hline Sex & $22 / 8$ & $20 / 10$ & \\
\hline Height & $167.67 \pm 7.2$ & $168 \pm 6.5$ & 0.653 \\
\hline Weight & $68.93 \pm 6.78$ & $67.9 \pm 6.05$ & 0.535 \\
\hline
\end{tabular}

As regards the PGIC score there was no statistically significant difference between the two groups $(P>0.05)$. The improvement in PGIC score from the pre-procedure was statistically significant in both groups $(P<0.01)$ (Table 5).

Table 2 Visual Analog Scale (VAS) Score

\begin{tabular}{|c|c|c|c|}
\hline & $\begin{array}{l}\text { Group S } \\
\text { (Mean士SD) }\end{array}$ & $\begin{array}{l}\text { Group F } \\
\text { (Mean士SD) }\end{array}$ & $P$ value \\
\hline Pre-procedure & $78.37 \pm 6.63$ & $78.4 \pm 5.56$ & 0.983 \\
\hline Ist week & $25.7 \pm 7.5$ & $35.23 \pm 9.5$ & $<0.01$ \\
\hline 4th week & $23.5 \pm 5.9$ & $33.3 \pm 7.7$ & $<0.01$ \\
\hline 8th week & $25.6 \pm 6.8$ & $32.43 \pm 7.56$ & $<0.01$ \\
\hline I2th week & $34.7 \pm 8.1$ & $37.8 \pm 7.01$ & 0.122 \\
\hline
\end{tabular}


Table 3 Duration of the Procedure and X-Ray Exposure

\begin{tabular}{|c|c|c|c|}
\hline & $\begin{array}{l}\text { Group S } \\
\text { (Mean士SD) }\end{array}$ & $\begin{array}{l}\text { Group F } \\
\text { (Mean士SD) }\end{array}$ & $P$ value \\
\hline $\begin{array}{l}\text { Duration procedure } \\
\text { (min) }\end{array}$ & $17.33 \pm 3.166$ & $30 \pm 6.4$ & $<0.01$ \\
\hline X-ray exposure (s) & $22.23 \pm 5.9$ & $96.6 \pm 32.7$ & $<0.01$ \\
\hline
\end{tabular}

Table 4 Satisfactory Score

\begin{tabular}{|c|c|c|c|}
\hline & $\begin{array}{l}\text { Group S } \\
\text { (Mean士SD) }\end{array}$ & $\begin{array}{l}\text { Group F } \\
\text { (Mean士SD) }\end{array}$ & $P$ value \\
\hline Pre-procedure & $1.57 \pm 1.165$ & $1.53 \pm 1.074$ & 0.909 \\
\hline Ist week & $7 \pm 1.05$ & $5.47 \pm 1.01$ & $<0.001$ \\
\hline 4th week & $7.07 \pm 1.08$ & $5.5 \pm 1.08$ & $<0.001$ \\
\hline 8th week & $9.7 \pm 1.3$ & $5.07 \pm 1.2$ & $<0.001$ \\
\hline I2th week & $5.43 \pm 1.07$ & $3.1 \pm 1.16$ & $<0.001$ \\
\hline
\end{tabular}

Table 5 Patient Global Impression of Change (PGIC) Score

\begin{tabular}{|l|l|l|l|}
\hline & $\begin{array}{l}\text { Group S } \\
\text { (Median } \\
\text { (Range)) }\end{array}$ & $\begin{array}{l}\text { Group F } \\
\text { (Median } \\
\text { (Range)) }\end{array}$ & P value \\
\hline Pre-procedure & $4(2-5)$ & $4.50(2-5)$ & 0.672 \\
Ist week & $2(I-4)$ & $2(I-4)$ & 0.542 \\
4th week & $2(I-4)$ & $2(I-4)$ & 0.407 \\
8th week & $2.50(I-4)$ & $3(I-4)$ & 0.868 \\
12th week & $3.50(2-5)$ & $4(2-5)$ & 0.649 \\
\hline
\end{tabular}

As regards the quality of life score there was no statistically significant difference between the two groups $(P>0.05)$. The improvement in quality of life score from the pre-procedure was statistically significant in both groups at the 1 st, 4 th, and 8 th week $(P<0.05)$ but not statistically significant at the 12 th week (Table 6).

There was no statistically significant difference between the two groups as regards the pre-procedure morphine

Table 6 Quality of Life

\begin{tabular}{|l|l|l|l|}
\hline & $\begin{array}{l}\text { Group } \\
\text { S (Median } \\
\text { (Range)) }\end{array}$ & $\begin{array}{l}\text { Group } \\
\text { F (Median } \\
\text { (Range)) }\end{array}$ & P value \\
\hline Pre-procedure & $2(0-4)$ & $2(0-4)$ & 0.899 \\
Ist week & $3(2-6)$ & $3(I-5)$ & 0.162 \\
4th week & $3(2-5)$ & $3(I-5)$ & 0.105 \\
8th week & $3(2-5)$ & $2(I-5)$ & 0.064 \\
12th week & $2(I-3)$ & $2(I-3)$ & 0.72 \\
\hline
\end{tabular}

consumption ( $P=0.407)$; however, there was a statistically significant difference in morphine consumption between the two groups at the 1st, 4 th, and 8 th week $(P<0.05)$ whereas at the 12 th week there is no significant difference $(P=0.132)$. The decline in morphine consumption from the preprocedure values was statistically significant in both groups and more significant in the group $\mathrm{S}(P<0.05)$ (Table 7).

Considering the adverse effects, 2 patients of group $\mathrm{S}$ and 3 patients of group $\mathrm{F}$ developed post-procedure hypotension, managed by IV fluids. There was a statistically significant difference between the two groups as regards position discomfort $(P<0.001)$. Needle discomfort and paresthesia did not occur in any patient of group $\mathrm{S}$ while it was reported in 4 patients in group F $(P<0.001)$. Nerve injury occurred in 2 patients in each group with transient mono and paraparesis which relieved after 2 to 3 weeks. No back pain was reported in group $\mathrm{S}$ patients while 11 patients of group $\mathrm{F}$ complained from post-procedure back pain $(P<0.001)$ (Table 8$)$.

\section{Discussion}

This work is to compare fluoroscopy alone against fluoroscopy integrated with ultrasound to guide SHB in pelvic cancer pain. The suggested integrated technique could have many advantages such as: the standard fluoroscopic posterior

Table 7 Morphine Dose (mg)

\begin{tabular}{|c|c|c|c|}
\hline & $\begin{array}{l}\text { Group S } \\
\text { (Mean } \pm \text { SD) }\end{array}$ & $\begin{array}{l}\text { Group F } \\
\text { (Mean士SD) }\end{array}$ & $P$ value \\
\hline Pre-procedure & $119 \pm 22.95$ & $114 \pm 23.43$ & 0.407 \\
\hline Ist week & $60.67 \pm 14.13$ & $74 \pm 15.45$ & 0.001 \\
\hline 4th week & $63.33 \pm 13.98$ & $73 \pm 15.12$ & 0.013 \\
\hline 8th week & $65 \pm 13.58$ & $77 \pm 16$ & 0.003 \\
\hline I2th week & $78 \pm 15.18$ & $84 \pm 15.22$ & 0.132 \\
\hline
\end{tabular}

Table 8 Adverse Effects (Number of Patients)

\begin{tabular}{|l|l|l|l|}
\hline & $\begin{array}{l}\text { Group S } \\
\text { (No.) }\end{array}$ & $\begin{array}{l}\text { Group F } \\
\text { (No.) }\end{array}$ & P value \\
\hline Hypotension & 2 & 3 & 0.865 \\
\hline Position discomfort & 0 & 12 & $<0.001$ \\
\hline $\begin{array}{l}\text { Needle discomfort } \\
\text { (paresthesia) }\end{array}$ & 0 & 4 & $<0.001$ \\
\hline Nerve injury & 2 & 2 & 1.00 \\
\hline Back pain & 0 & 11 & $<0.001$ \\
\hline
\end{tabular}


view is performed in relation to the spinal column, which in many cases is the site of severe spondylo-degenerative changes especially at L5/S1 level (eg, degenerated disc with worked loss of L5/S1 discal height that hinders transdiscal approach and high iliac crest, enlarged L5 transverse process, annular disc fracture forming osteophytes, partial and/or complete unilateral or bilateral Bertolotti's syndrome, etc) which all interfere with postero-lateral trajectory of SHB. Furthermore, the L4 level paramedian approach is time consuming, traumatic, and troublesome as inducing many possible visceral and vascular injuries, besides the installed neurolytic agent is usually away from the proper site of SHB, namely the L5/S1 junction, paramedian, and in front of the sacral promontory. Thus, the only hope in these cases of difficult posterior fluoroscopic approach is the anterior ultrasound proposed technique.

In addition, U/S guided techniques have a lot of benefits like bedside facility, less time consumption, better tolerability in frail cancer patients with poor general conditions, and less radiation exposure. Also, the final position of the needle tip and subsequently neurolytic drug demand, the fluoroscopic precision of the L5/S1 paramedian approach, position (at $\mathrm{P} / \mathrm{A}$ projection) and smooth contour anterior to the psoas fascia (at lateral projection) away from the L5 nerve root, and epidural, subdural, or intrathecal spread of contrast media and neurolytic drug.

Also, the integrated guidance protocol is widely applied in the field of pain intervention such as biplanar (fluoroscopy/CT scan) for vertebroplasty, treatment of trigeminal neuralgia, percutaneous cervical cordotomy (PCC), etc. The integrated guidance technique is of paramount benefit in such neurolytic block of SHB (not amenable to neuroablative therapy of thermo-coagulative RF) particularly in pain patients with disturbed pelvic anatomy (post-surgical, post-radiotherapy). The combination of U/S with standard fluoroscopy is of much less radiation exposure than the CT scan. Hence, this study is the first trial to use ultrasound assisted by fluoroscopy for SHPB, and it was found that this technique is less time consuming, with less X-ray exposure in comparison to the oblique fluoroscopic guided technique $(P<0.01)$. The authors also found that the anterior ultrasound guided SHPB assisted by fluoroscopy provided a marked decrease in VAS score and morphine consumption and it is superior to the oblique guided fluoroscopic technique $(P<0.01)$ with no difference between the techniques at 12 weeks $(P=0.122)$. Unlike the previous study, which was limited by the lack of absolute confirmation of intravascular uptake and chances of bowel and urinary bladder injury, ${ }^{7}$ the use of two modalities at the same time in our study overcame this limitation.

Although opioids remain the cornerstone of cancer pain treatment, $^{13}$ it is known that pelvic cancer patients may experience severe pain that is resistant to oral or parenteral opioids. In addition, excessive sedation or other side effects may limit the acceptability and usefulness of oral or parenteral opioid therapy. Therefore, interventional techniques may be needed to control pain and improve the quality of life of these patients. The superior hypogastric plexus neurolysis represents a reproducible and effective alternative in the management of pain in patients with pelvic cancer. ${ }^{6,13-15}$

Plancarte et al was the first to describe SHPB using neurolytics and bilateral needles, $t$ reporting $70 \%$ of pain reduction. ${ }^{16}$ That classic approach aided by fluoroscopy described by the same author had been reported and resulted in $72 \%$ satisfactory pain relief (VAS $<4 / 10$ ) with $43 \%$ decrease in opioid consumption. ${ }^{3}$

Although a single case series in addition to a randomized controlled trial of ultrasound guided SHPB has been reported in the literature, ${ }^{7}$ a randomized, controlled study of anterior US guided (SHPB) assisted by fluoroscopy has not been so far documented.

The study by Mishra et al using anterior ultrasound guided SHPB in advanced gynecological malignancy, in comparison with control group receiving opioid and adjuvant treatment, found that the technique provided a marked decrease in VAS score and morphine consumption $(P<0.05)$, and there was no significant difference between the two groups $(P=0.586)$ for VAS scores at 3 month follow up. ${ }^{7}$ In this study, the improvement in VAS score was statistically significant in both groups in comparison with the preblock values $(P<0.05)$, but there was a difference between the groups throughout the study period.

Similar results related to the performance scale for QOL assessment and adverse effects were observed by other authors. ${ }^{10}$

Another study by Kamel et al comparing fluoroscopic guided versus anterior ultrasound guided SHPB showed pain relief in both groups with no significant difference, also there was improvement in PGIC, patient satisfaction score (PSS), and opioid consumption from the preprocedure values with no significant difference between the two groups. ${ }^{12}$

Kawamata et al evaluated the effectiveness of celiac block on QOL and pain relief with traditional morphine and NSAID treatment. ${ }^{11}$ It was concluded that celiac block does not directly improve QOL in pancreatic cancer but it 
could stop deterioration in QOL in comparison with morphine-NSAID by its long-lasting analgesia and the limitation of adverse effects from the decrease in morphine consumption. $^{11}$

Possible indications and different pain mechanisms for SHPB in 22 pelvic cancer patients were studied. ${ }^{17}$ It concluded that fluoroscopic guided SHPB has to be considered as an adjuvant technique and not primary treatment in consideration of the different pain mechanisms involved in malignancies. Although the usage of opioids is generally associated with side effects, they remain the mainstay of management of pain related to malignancy. The selective technique of intervention is required only in $10-15 \%$ of the cancer patients with severe pain resistant to traditional opioid and co-analgesic therapies. ${ }^{17}$

Similarly, in the studies by Mishra et $\mathrm{al}^{7}$ and Kamel et al, ${ }^{12}$ using ultrasound guided SHPB, they concluded that this technique has potential drawbacks. There is the potentiality of injury to structures overlying and surrounding the SHP such as the lumbo-sacral plexus, bladder, ureters, uterus, bowed and iliac vessels, and the risk of infection from perforating the bowel.

Contrarily, similar precautions were taken by the authors to avoid such drawbacks. Pre-procedural bladder and bowel preparation, Trendelenburg position, and smaller sized Chiba needle can avoid injury of the viscera, as collapsed viscera tend to fall away from the pathway of the needle. To avoid vascular injury, we used the color Doppler mode followed by aspiration and then injection of dye to see the distribution and the run-off phenomenon under fluoroscopy.

In the study by Kamel et al using ultrasound guided SHPB, they mentioned that this technique is bed-side, fast, and comfortable to the patient, as a supine position is favorable for generally ill patients who cannot lie prone. It decreases the discomfort due to passing the needles through muscles of the back and avoids L5 nerve root injury. ${ }^{12}$ It may be also the only applicable technique in older patients with advanced spondylodegenerative changes of the spine in which the fluoroscopic guidance posterior techniques (oblique, trans-discal) could not be performed. ${ }^{12}$

Limitations of this study are the possibility of bowel or bladder injury and lumbo-sacral neurological deficits due to unpredicted and uncontrolled spread of the neurolytic drug. Also, a small sample size, the patient cannot be blinded (due to the nature of the intervention) which may be a source of bias, the need for larger meta-analysis to verify results, and longer follow up periods.

\section{Conclusion}

This study showed that the anterior ultrasound guided SHPB aided by fluoroscopy is more superior to the standard fluoroscopic guided technique in relieving pain and in decreasing the morphine consumption. It is a simple technique with minimal discomfort to the patient as it is done in the supine position, making it more helpful for generally ill cancer patients who cannot lie prone. It is also less time consuming, offering less exposure to radiation and fewer drawbacks.

\section{Disclosure}

The authors declare no conflicts of interest.

\section{References}

1. Apte G, Nelson P, Brismée JM, Dedrick G, Justiz R, Sizer PS. Chronic female pelvic pain-part 1: clinical pathoanatomy and examination of the pelvic region. Pain Pract. 2012;12(2):88-110. doi:10.1111/j.1533-2500.2011.00465.x

2. Hanno PM, Burks DA, Clemens JQ, et al. AUA Guideline for the diagnosis and treatment of interstitial cystitis/bladder pain syndrome. J Urol. 2011;185:2162-2170. doi:10.1016/j.juro.2011.03.064

3. Plancarte R, De-leon-casasola OA, El-Helaly M, Allend S, Lema MJ. Neurolytic superior hypogastric plexus block for chronic pelvic pain associated with cancer. Reg Anesth. 1997;22:562-568.

4. Cleeland C, Gonin R, Hatfield A, et al. Pain and its treatment in outpatients with metastatic cancer. $N$ Engl J Med. 1994;330:592-596. doi:10.1056/NEJM199403033300902

5. Prithvi Raj P, Erdine S. Pain-relieving procedures: the illustrated guide. In: Chapter 17: Interventional Pain Procedures in the Pelvic and Sacral Regions. 1st edition. John Wiley and sons Ltd; 2012:365-371.

6. Ayman AG, Sahar MM. Comparative study between CT guided superior hypogastric plexus block and classic posterior approach: a prospective randomized study. Saudi J Anesth. 2014;8 (3):378-383. doi:10.4103/1658-354X.136625

7. Mishra S, Bhatnagar S, Rana SP, Khurona D, Thulkar S. Efficacy of the anterior ultrasound guided superior hypogastric plexus neurolysis in pelvic cancer pain in advanced gynecological cancer patients. Pain Med. 2013;14:837-842. doi:10.1111/pme.12106

8. Choi JW, Kim WH, Lee CJ, Sim WS, Park S, Chae HB. The optimal approach for a superior hypogastric plexus block. Pain Pract. 2018;18(3):314-321. doi:10.1111/papr.12603

9. Alexandra Junewicz SJ, Youngner SJ. Patient-satisfaction surveys on a scale of 0 to 10: improving health care, or leading it astray? Hastings Center Rep. 2015;45:43-51. doi:10.1002/hast.453

10. Dworkin RH, Turk DC, Wyrwich KW, et al. Interpreting the clinical importance of treatment outcomes in chronic pain clinical trials: IMMPACT recommendations. $J$ Pain. 2008;9(2):105-121. doi:10.1016/j.jpain.2007.09.005

11. Kawamata M, Ishitani K, Ishikawa K, et al. Comparison between celiac plexus block and morphine treatment on quality of life in patients with pancreatic cancer pain. Pain. 1996;64:597-602. doi:10.1016/0304-3959(95)00189-1

12. Kamel MA, Ahmed AR, Shaaban MH, Hashem RH. Comparison between fluoroscopic posterior versus ultrasound-guided anterior approach for superior hypogastric plexus neurolysis: a prospective, randomized, comparative study. Res Opin Anesth Intensive Care. 2016;3:151-156. doi:10.4103/2356-9115.195882 
13. Rocha A, Plancarte R, Nataren RGR, Carrera IHS, Pacheco VALR, Hernandez-Porras BC. Effectiveness of superior hypogastric plexus neurolysis for pelvic cancer pain. Pain Physician. 2020;23 (2):203-208.

14. De Leon-casasola OA, Kent E, Lema MJ. Neurolytic superior hypogastric plexus block for chronic pelvic pain associated with cancer. Pain. 1993;54:145-151. doi:10.1016/0304-3959(93)90202-Z

15. Schmidt AP, Schmidt SR, Riberio SM. Is superior hypogastric plexus block effective for treatment of chronic pelvic pain? Rev Bras Anesthesiol. 2005;55:669-679. doi:10.1590/S0034-70942005 000600011
16. Plancarte R, Amescua C, Patt RB, Aldrete JA. Superior hypogastric plexus block for pelvic cancer pain. Anesthesiology. 1990;73 (2):236-239. doi:10.1097/00000542-199008000-00008

17. Mercadante S, Fulfaro F, Casuccio A. Pain mechanisms involved and outcome in advanced cancer patients with possible indication for celiac plexus block and superior hypogastric plexus block. Tumori. 2002;88:243-245. doi:10.1177/030089160208800311

\section{Publish your work in this journal}

The Journal of Pain Research is an international, peer reviewed, open access, online journal that welcomes laboratory and clinical findings in the fields of pain research and the prevention and management of pain. Original research, reviews, symposium reports, hypothesis formation and commentaries are all considered for publication. The manuscript

Submit your manuscript here: https://www.dovepress.com/journal-of-pain-research-journa management system is completely online and includes a very quick and fair peer-review system, which is all easy to use. Visit http:// www.dovepress.com/testimonials.php to read real quotes from published authors. 